\title{
Possible Spin-Liquid States on the Triangular and Kagomé Lattices
}

\author{
Kun Yang, L. K. Warman, and S. M. Girvin \\ Physics Department, Indiana University, Bloomington, IN 47405
}

\begin{abstract}
The frustrated quantum spin-one-half Heisenberg model on the triangular and Kagomé lattices is mapped onto a single species of fermion carrying statistical flux $\theta=\pi$. The corresponding Chern-Simons gauge theory is analyzed at the Gaussian level and found to be massive. This provides a new motivation for the spin-liquid Kalmeyer-Laughlin wave function. Good overlap of this wave function with the numerical ground state is found for small clusters.

PACS numbers: 75.10.Jm, 75.50.Ee, 73.20.Dx
\end{abstract}

Typeset Using REVTEX 
The 2D spin- $\frac{1}{2}$ antiferromagnetic Heisenberg model has attracted a lot of interest over the last a few years. It is widely believed that the ground state has long-range Néel order on the square lattice. ${ }^{1}$ On the triangular and Kagomé lattices, the situation is much less clear due to the geometric frustration. In the case of triangular lattice, Huse and Elser have constructed variational wave functions with long-range order and low energies. ${ }^{2}$ Numerical evidence was found supporting this scenario. ${ }^{3}$ Recently however, Singh and Huse ${ }^{4}$ have calculated the sublattice magnetization using a series expansion method, which is believed to be accurate, and found that the ground state is nearly disordered for the triangular lattice and strongly disordered for the Kagomé lattice due to the large degeneracy of classical ground state configurations. ${ }^{5}$

Recently a two-dimensional extension of the Jordan-Wigner transformation, which essentially treats hard core-bosons (see below) as fermions with flux tubes, or equivalently, fermions coupled to a Chern-Simons gauge field, was developed. ${ }^{6}$ The advantage of this approach is that the unwanted hard-core condition in the boson picture is taken care of by the Pauli principle, but as a price one has to introduce gauge interactions to fix the statistics. We applied this method to both triangular and Kagomé lattices. At mean field level, the flux carried by the fermions is smeared out to form a uniform background magnetic field. If we neglect the Ising part of the Hamiltonian (which becomes a nearest-neighbor repulsive interaction between fermions after the transformation) for the moment, we have noninteracting fermions moving in a constant magnetic field. Numerical diagonalization yields two and six Landau subbands, with large excitation gaps ${ }^{23}$ at the Fermi level of $2 \sqrt{3} J$ and $1.46 J$ for the triangular and Kagomé lattices respectively ( $J$ is the coupling between neighboring spins). The Hall conductance is quantized in such cases, in the form $\sigma_{x y}=\frac{e^{2}}{h} \nu$ where $e$ is the charge carried by the fermions. In the continuum $\nu$ is just the Landau level filling factor $\nu=\frac{\pi}{\theta}$, where $\theta$ is the statistics angle which is $\pi$ in this case. However on a lattice, $\nu$ is one of the $\operatorname{TKKN}^{7}$ integers, not necessarily equal to $\frac{\pi}{\theta}$. If one examines Gaussian fluctuations of the gauge field about its saddle point (mean field), one can integrate out the fermion degrees of freedom (which are quadratic in the action) and expand the effective action for the gauge 
field about its saddle point to second order to obtain ${ }^{8}$

$$
\begin{aligned}
S\left[A_{\mu}\right] & =S_{0}+\int d^{2} x d t\left[\frac{\epsilon}{2} E^{2}(\vec{x}, t)-\frac{\chi}{2} B^{2}(\vec{x}, t)\right] \\
& +\frac{1}{4} \int d^{2} x d t\left(\nu-\frac{\pi}{\theta}\right) \epsilon_{\mu \nu \lambda} A^{\mu} F^{\nu \lambda}+\cdots,
\end{aligned}
$$

where $S_{0}$ is the mean field action, $\epsilon$ and $\chi$ are the mean field values of the long-wavelength, low-frequency dielectric constant and diamagnetic susceptibility respectively. As noted by Fradkin, ${ }^{8}$ the fluctuation is massless if and only if the Chern-Simons term in the action is cancelled, i.e., $\nu=\frac{\pi}{\theta}$. This is easy to understand in terms of self-consistent linear response. Assume there is a long-wave length, low-frequency fluctuation of the density of the fermions. Since the fermions carry flux, there should be a fluctuation of magnetic field in the same mode. According to Maxwell's equations, there will be a nonzero line integral of electric field around any region $\Gamma$ :

$$
\oint_{\Gamma} \vec{E} \cdot d \vec{l}=-\frac{1}{c} \frac{d \Phi}{d t}=-\frac{h}{e} \frac{\theta}{\pi} \frac{d N}{d t},
$$

where $\Phi$ and $N$ are the flux and number of particles in that region respectively. Now look at the response of $N$ to this electric field:

$$
\frac{d Q}{d t}=e \frac{d N}{d t}=-\oint_{\Gamma} \sigma_{x y} \vec{E} \cdot d \vec{l}=-\frac{\sigma_{x y}}{c} \frac{d \Phi}{d t} .
$$

We see the above equations are consistent if and only if $\nu=\frac{\pi}{\theta}$. This is guaranteed in the continuum, where Fetter et al did find a gapless collective mode. ${ }^{19}$ We have computed the TKKN integer for the triangular and Kagomé lattices using the method of MacDonald ${ }^{20}$ and found in both cases $\nu=-1 \neq \frac{\pi}{\theta}=1$. Hence we expect the Chern-Simons field to be massive and the quantum XY model is therefore likely to have a gap assuming no broken translation symmetry. This gap may be stable against the Ising perturbation causing the Heisenberg model to have a spin liquid ground state on these lattices.

By making analogy to the fractional quantum Hall effect (FQHE), Kalmeyer and Laughlin $(\mathrm{KL})$ suggested a very interesting spin liquid wave function for the Heisenberg model on the triangular lattice. ${ }^{9,10}$ It was found to have reasonably good energy (about ten percent 
higher than the best numerical estimate). We believe the massiveness of the Chern-Simons theory demonstrated here provides a more fundamental motivation for quantum Hall physics in frustrated spin systems. Further more, within the single-mode approximation ${ }^{12}$ an excitation gap in a 2D system requires a Jastrow-like wave function whose square is a 2D one-component plasma in order for the structure factor to vanish at small $q: S(q) \sim q^{2}$. $\mathrm{SU}(2)$ symmetry uniquely restricts the coefficient of the plasma charge to be that given by the $\mathrm{KL}$ wave function $(m=2)$. The spin- $\frac{1}{2}$ excitations argued by Laughlin ${ }^{11}$ similarly require $m=2$ in the Bose representation. These arguments strongly suggest that a spin liquid wave function should be of the Kalmeyer-Laughlin type.

In the rest part of this paper we first briefly review the KL wave function, and prove that it is equivalent to a projected underlying fermion wave function. We apply this new wave function to the Kagomé net, and calculate its energy using the Monte Carlo method. Then we calculate the overlap between this wave function and the exact wave functions on small clusters. Finally we summarize and discuss our results.

The Hamiltonian for antiferromagnetic Heisenberg model is:

$$
H=J \sum_{<i j>} \vec{S}_{i} \cdot \vec{S}_{j}
$$

where $J>0, \vec{S}_{i}$ is the spin operator at site $i$, and the sum is over nearest neighbors. Following Ref[9], we map the spin operators to hard-core boson operators. The Hamiltonian in this representation is

$$
\begin{aligned}
H & =T+V \\
T & =\frac{J}{2} \sum_{<i j>}\left(a_{i}^{\dagger} a_{j}+a_{j}^{\dagger} a_{i}\right) \\
V & =J \sum_{<i j>} n_{i} n_{j}+\text { const }
\end{aligned}
$$

Here $N_{s}$ is the number of sites of the lattice, $a_{j}$ is the hard-core boson operator at $j$ th site. As was shown by $\mathrm{KL},{ }^{9}$ on the triangular lattice, after an appropriate gauge transformation, this new Hamitonian describes hard core bosons moving in a uniform magnetic field with field strength one flux quantum per unit cell, under the symmetric gauge. The bosons have a 
nearest-neighbor repulsive interaction besides the hard core condition. In the ground state, the number of bosons $N=N_{s} / 2$, which means the Landau level filling factor is one half. By making analogy to the FHQE, they suggested the trial wave function for the bosons:

$$
\Psi\left(z_{1}, \cdots, z_{N}\right)=\prod_{i<j}\left(z_{i}-z_{j}\right)^{2} \prod_{k \leq N} G\left(z_{k}\right) e^{-\frac{1}{4}\left|z_{k}\right|^{2} / l_{0}^{2}}
$$

Here $z_{j}=x_{j}+i y_{j}$ is the complex coordinate of the lattice site occupied by the $j$ th boson, $l_{0}=b(\sqrt{3} / 4 \pi)^{\frac{1}{2}}$ is the magnetic length which is set to 1 afterwards, $b$ is the lattice constant. $G\left(z_{k}\right)= \pm 1$ are gauge phases introduced by the gauge transformation. ${ }^{9}$

The wave function (2) has some nice features, including being a singlet state in the thermodynamic limit, but it can not be generalized to non-Bravais lattices, such as the Kagomé lattice. Also it becomes a singlet only when the system is infinitely large, so it is not suitable for finite size studies. For these reasons, we want to find a more general wave function that returns to (2) on the triangular lattice in the thermodynamic limit, and has better finite-size properties. To do that, we assume the spins are carried by spin- $\frac{1}{2}$ fermions that are trapped on lattice sites, and try to describe state (2) in terms of these underlying fermions. ${ }^{21}$ We can express the spin operators in terms of these fermion operators:

$$
\begin{aligned}
& a_{j}^{\dagger}=S_{j}^{+}=c_{j \uparrow}^{\dagger} c_{j \downarrow} \\
& a_{j}=S_{j}^{-}=c_{j \downarrow}^{\dagger} c_{j \uparrow}
\end{aligned}
$$

where $c_{j \uparrow, \downarrow}^{+}$are creation operators of up(down) spin fermions at $j$ th site. Just as for the bosons, there is a hard core condition on the fermions: $n_{j \uparrow}+n_{j \downarrow}=1$.

In the second quantized representation, the state (2) is

$$
|\Psi\rangle=\sum_{\left\{z_{1}, \cdots, z_{N}\right\}} \Psi\left(z_{1}, \cdots, z_{N}\right) a_{z_{1}}^{\dagger} \cdots a_{z_{N}}^{\dagger}\left|0_{b}\right\rangle
$$

Here the sum is over all possible boson configurations. $\left|0_{b}\right\rangle$ is the boson vacuum state. Since it corresponds to the state that all spins are down, we have

$$
\left|0_{b}\right\rangle=\prod_{j=1}^{N_{s}} c_{j \downarrow}^{\dagger}\left|0_{f}\right\rangle
$$


where $\left|0_{f}\right\rangle$ is the fermion vacuum state. So we get

$$
\begin{aligned}
|\Psi\rangle & =\frac{1}{N !} \sum_{\left\{z_{1}, \cdots, z_{N} ; z_{[1]}, \cdots, z_{[N]}\right\}} \Psi\left(z_{1}, \cdots, z_{N}\right) \\
& \times c_{z_{1 \uparrow}}^{\dagger} c_{z_{1 \downarrow}} \cdots c_{z_{N \uparrow}}^{\dagger} c_{z_{N \downarrow}} \prod_{k=1}^{N_{s}} c_{k_{\downarrow}}^{\dagger}\left|0_{f}\right\rangle .
\end{aligned}
$$

Here $z_{[j]}=z_{N+j}$ denotes the coordinate of $j$ th down spin fermion. The sum is over all possible fermion configurations satisfying the hard core condition. We do not distinguish $c_{j}$ and $c_{z}, a_{j}$ and $a_{z}$, etc., if $z$ is the complex coordinate of $j$ th site. Rearranging the order of fermion operators and neglecting constant factors, we have

$$
\begin{gathered}
|\Psi\rangle=\sum_{\left\{z_{1}, \cdots, z_{N} ; z_{[1]}, \cdots, z_{[N]}\right\}} \Psi\left(z_{1}, \cdots, z_{N}\right) \\
\times F\left(z_{1}, \cdots, z_{N} ; z_{[1]}, \cdots, z_{[N]}\right) \prod_{k=1}^{N} c_{z_{k \uparrow}}^{\dagger} \prod_{l=1}^{N} c_{z_{l \downarrow}}^{\dagger}\left|0_{f}\right\rangle .
\end{gathered}
$$

Here $F\left(z_{1}, \cdots, z_{[N]}\right)$ is a totally antisymmetric factor:

$$
\begin{aligned}
\left|F\left(z_{1}, \cdots, z_{2 N}\right)\right| & =\text { const } \\
F\left(\cdots, z_{j}, \cdots, z_{k}, \cdots, z_{2 N}\right) & =-F\left(\cdots, z_{k}, \cdots, z_{j}, \cdots\right) .
\end{aligned}
$$

We can take $F$ to be

$$
F=\prod_{i<j}\left(z_{i}-z_{j}\right)^{-1}\left(z_{[i]}-z_{[j]}\right)^{-1} \prod_{k, l \leq N}\left(z_{k}-z_{[l]}\right)^{-1}
$$

If we go back to first quantization, the wave function that describes the underlying fermions is just

$$
\begin{aligned}
& \Psi_{f}\left(z_{1}, \cdots, z_{[N]}\right)=\prod_{i<j \leq N}\left(z_{i}-z_{j}\right)\left(z_{[i]}-z_{[j]}\right)^{-1} \\
\times & \prod_{k, l \leq N}\left(z_{k}-z_{[l]}\right)^{-1} \prod_{m=1}^{N} G\left(z_{m}\right) e^{-\frac{1}{4}\left|z_{m}\right|^{2}} .
\end{aligned}
$$

Obviously a fermion wave function should be antisymmetric under exchange (including spin variables), but here we neglect spin variables in the wave function and treat up and down spin particles as if they were distinguishable, so the above wave function is legal. ${ }^{18}$ 
Since we have the hard core condition on fermions, what we really mean by (3) is the state that is projected to the subspace with no double occupancy. Hence it is a well defined wave function for the original quantum spins.

Now we can use a theorem proved by $\mathrm{KL}^{10}$ :

$$
\prod_{j \neq k}\left(\xi_{k}-\xi_{j}\right)=C_{0} G\left(\xi_{k}\right) e^{\frac{1}{4}\left|\xi_{k}\right|^{2}}
$$

where $\xi_{j}$ is the complex coordinate of $j$ th site, $C_{0}$ is a constant,and $G\left(\xi_{k}\right)$ are the gauge phases. ${ }^{9}$ This theorem holds only on the triangular lattice in the thermodynamic limit. Using it in (3) we get up to a constant factor,

$$
\Psi_{f}\left(z_{1}, \cdots, z_{[N]}\right)=\prod_{i<j \leq N}\left(z_{i}-z_{j}\right)\left(z_{[i]}-z_{[j]}\right)
$$

The right hand side of equation(4) is just the product of two Vandermonde determinants, ${ }^{21}$ which is what one gets when both spin states of the first Landau level are fully occupied. Since up and down spin particles occupy the same spatial Slater determinant, the resulting state must be a spin singlet, even after projection. ${ }^{16}$ This provides another way to prove that the state (2) is a singlet in the thermodynamic limit. The advantage of (4) is it gives a singlet even on finite size systems, and it can be generalized directly to non-Bravais lattices.

As a test of the equivalence between (2) and (4), we calculated the energy of (4) on a triangular lattice. The calculation of the Ising part of the energy is straightforward using Monte Carlo, and the total energy is exactly three times that (for any system size). The extrapolated result is $-0.48 \pm 0.01 J$ per site, which agrees with the KL result. ${ }^{9}$ We have found that in our case the data converges much faster, i.e., the finite size results are much closer to the extrapolated result. This tells us that (4) is better for finite size study. The energy we get for the Kagomé is $-0.399 \pm 0.001 \mathrm{~J}$ per site, about eight percent higher than the best numerical estimate. ${ }^{15}$

We have also studied the overlap between (4) and exact ground state on small clusters, where we need to minimize the finite size effect by applying periodic boundary conditions 
(PB). The wave function (4) satisfies open boundary condition, so we need to solve the wave functions with PB:

$$
t\left(\overrightarrow{L_{j}}\right) \psi(z)=e^{i \phi_{j}} \psi(z) ; j=1,2, \cdots
$$

Here $t\left(\overrightarrow{L_{j}}\right)$ is a magnetic translation operator. ${ }^{13}$ This problem was solved for the torus geometry by Haldane and Rezayi. ${ }^{13}$ It turns out that the filled Landau level wave function, after neglecting constant factors, can be expressed in terms of elliptic theta functions ${ }^{13,14}$ :

$$
\begin{aligned}
& \Psi_{f}=\prod_{i, j \leq N}\left[\theta_{1}\left(\frac{\pi}{L_{1}}\left(z_{i}-z_{j}\right) \mid \tau\right) \theta_{1}\left(\frac{\pi}{L_{1}}\left(z_{[i]}-z_{[j]}\right) \mid \tau\right)\right] \\
& \times \theta_{1}\left(\frac{\pi}{L_{1}}\left(\sum_{k} z_{k}-Z_{0}\right) \mid \tau\right) \theta_{1}\left(\frac{\pi}{L_{1}}\left(\sum_{k} z_{[k]}-Z_{0}\right) \mid \tau\right) .
\end{aligned}
$$

Here $\theta_{1}(z \mid \tau)$ is the elliptic theta function, $\tau=L_{2} e^{i \delta} / L_{1}, \overrightarrow{L_{1}}$ and $\overrightarrow{L_{2}}$ are the vectors that determine the shape of the parallelogram, and $\delta$ is the angle between them. $Z_{0}$ is the center of mass coordinate which is determined by $\phi_{1}$ and $\phi_{2}$. In most cases we are interested in, it should be set to zero. ${ }^{17}$ Like eq. (4), eq. (6) also describes a singlet state. A truely nondegenerate ground state wave function must be real. ${ }^{9}$ So instead of using the complex wave function (6) directly, we use $\Psi_{f} e^{i \phi}+\Psi_{f}^{*} e^{-i \phi}$ in the overlap calculation and use $\phi$ as a variational parameter. We applied it to several clusters of the triangular lattice with the shape of a parallelogram (torus geometry) and one with the shape of a hexagon (twelve spins). The results are listed in table 1 . We have found that the square of the overlap remains large in systems with up to twenty spins $(4 \times 5)$. The energies one gets are close to the Monte Carlo result, which means the change of boundary condition does not change the short distance correlations. For reasons we do not understand yet, the overlap is exactly zero in the $4 \times 4$ cluster. We have verified that $\Psi_{f}$ has the correct symmetries (spin rotation, translation, $180^{\circ}$ rotation and mirror reflection of space). Apparently there is some additional hidden symmetry which does not match that of the numerical ground state. We have done the same calculations on Kagomé clusters ${ }^{15}$ with twelve and eighteen spins. Again we got zero overlaps, probably for similar reasons. The energies, $-0.420 J$ and $-0.418 J$ per site for twelve- and eighteen-spin clusters respectively, are close to the Monte Carlo result. 
If the ground state of the triangular lattice has three sublattice order, such order is suppressed on clusters $3 \times 4,4 \times 4$ and $4 \times 5$ due to incommensurability, but is not on $6 \times 3$ and the hexagon with twelve spins. Our data suggests this commensurability is a weak effect. Both state (6) and the true ground states have a lot of symmetries (rotation, translation, etc.). It could happen that there are so few states of the right symmetries available that an arbitrary combination of them will have a decent overlap with the ground state. By assuming singlet states are uniformly distributed in the momentum space, we find the number of states with the right symmetry is of order 1000 in the case of twenty spins, and yet the square of the overlap is rather large: 0.493. The twelve-spin hexagon has additional symmetries, so the significance of the remarkably large squared overlap of 0.966 is unclear.

Quantum Hall wave functions exhibit hidden ODLRO due to the binding of vortices to charges. $^{24}$ This corresponds to chiral order in the present problem. ${ }^{25}$ The large overlaps we obtain suggest chiral order is present in the exact ground states of the clusters.

The central result of this paper is the demonstration that treating spins as fermions carrying flux tubes leads to a massive Chern-Simons theory on frustrated lattices. This provides a new and fundamental motivation for quantum Hall types of spin-liquid physics. We have developed the formalism needed to compute the overlap between these wave functions and the exact ground state and we have obtained significant overlaps for small clusters. It would be highly desirable to see these calculations extended to large lattices, although this will require considerable numerical effort.

We acknowledge helpful discussions with Rajiv Singh, V. Elser, A. J. Berlinsky, C. Kallin and N. Read. This work was supported by NSF-9113911. 


\section{REFERENCES}

[1] E. Manousakis, Rev. Mod. Phys. 63, 1 (1991).

[2] D. Huse and V. Elser, Phys. Rev. Lett. 60, 2531 (1988).

[3] B. Bernu et al, Phys. Rev. Lett. 69, 2590 (1992).

[4] R. Singh and D. Huse, Phys. Rev. Lett. 68, 1776 (1992).

[5] See, for example, A. Harris et al, Phys. Rev. B 45, 2899 (1992); S. Sachdev, Phys. Rev. B 45, 12377 (1992); J. Chalker and J. Eastmond, preprint (1992); see, however, A. Chubukov, Phys. Rev. Lett. 69, 832 (1992).

[6] E. Fradkin, Phys. Rev. Lett. 63, 322 (1989); Y. R. Wang, Phys. Rev. B 43, 3786 (1991).

[7] D. J. Thouless et al, Phys. Rev. Lett. 49, 405 (1982).

[8] E. Fradkin, Phys. Rev. B 42, 570 (1990).

[9] V. Kalmeyer and R. B. Laughlin, Phys. Rev. Lett. 59, 2095 (1987).

[10] V. Kalmeyer and R. B. Laughlin, Phys. Rev. B 39, 11879 (1989).

[11] R. B. Laughlin, Science 242, 525 (1988).

[12] S. M. Girvin et al, Phys. Rev. Lett. 54, 581 (1985); Phys. Rev. B 33, 2481 (1986).

[13] F. D. M. Haldane and E. H. Rezayi, Phys. Rev. B 31, 2529 (1985).

[14] I. S. Gradshteyn and I. M. Ryzhik, Tables of Integrals, Series and Products (Academic, New York, 1980), p. 921.

[15] C. Zeng and V. Elser, Phys. Rev. B 42, 8426 (1990).

[16] This can be proved using the Fock cyclic conditions. ${ }^{18}$

[17] Except for the hexagon case discussed later, where we need a non zero $Z_{0}$ so that (6) has the right symmetry. 
[18] D. Yoshioka et al, Phys. Rev. B 38, 3636 (1988).

[19] A. Fetter et al, Phys. Rev. B 39, 9679 (1989).

[20] A. H. MacDonald, private communication. Suppose one has solved the mean field fermion spectrum and found there are $n$ bands below the Fermi energy. Then one increases the flux of each magnetic unit cell by a small amount $\frac{\Phi_{0}}{N}$, so the new magnetic unit cell is $N$ times as large as before, and each band will split into $N$ subbands. After solving the new spectrum one finds there are $m$ subbands below the Fermi energy. According to Středa formula, ${ }^{22} \nu=m-N n$.

[21] Similar results have been obtained before. See R. B. Laughlin and Z. Zou, Phys. Rev. B 41, 664 (1990); E. J. Mele and D. C. Morse, Phys. Rev. B 42, 150 (1990). We believe the proof presented here is more explicit and straightforward.

[22] Středa, J. Phys. C 15, L717 (1982).

[23] Y. R. Wang [Phys. Rev. B 45, 12604 (1992)] uses a staggered mean field with zero net flux per unit cell on the triagular lattice and hence obtains only a single band with no gap. We believe this is incorrect.

[24] S. M. Girvin, in the Appendix of The Quantum Hall Effect, 2nd edition, editted by R. E. Prange and S. M. Girvin (Springer, New York, 1990).

[25] X. G. Wen et al, Phys. Rev. B 39, 11413 (1989) 


\section{TABLES}

TABLE I. overlaps and variational energies on small clusters of the triangular lattice

\begin{tabular}{cccc}
\hline \hline cluster & $\mid$ overlap $\left.\right|^{2}$ & $E_{v}$ & exact energy \\
\hline hexagon(12) & 0.966 & -0.591 & -0.6103 \\
$3 \times 4$ & 0.821 & -0.519 & -0.5776 \\
$4 \times 4$ & 0.000 & -0.459 & -0.5347 \\
$6 \times 3$ & 0.554 & -0.491 & -0.5811 \\
$4 \times 5$ & 0.493 & -0.481 & -0.5581 \\
\hline \hline
\end{tabular}

Energies are in unit $J$ per site; $E_{v}$ is the variational energy. 